\title{
Radiation exposure levels in close proximity to therapeutic patients treated for thyroid carcinoma with high dose I-131
}

\author{
${ }^{1}$ Ferdoushi Begum, ${ }^{1}$ Nurun Nahar, ${ }^{1}$ Kamila Afroj Quadir, ${ }^{1}$ Jasmine Ara Haque, ${ }^{1}$ Md. Nahid \\ Hossain, ${ }^{1}$ Md. ${ }^{1}$ Simoon Salekin, ${ }^{1}$ Afroza Parvin \\ ${ }^{1}$ National Institute of Nuclear Medicine \& Allied Science, Dhaka, \\ ${ }^{2}$ Institute of Nuclear Medicine \& Allied Science, Mitford
}

\begin{abstract}
Address for Correspondence: Ferdoushi Begum, Principal Scientific officer National Institute of Nuclear Medicine \&Allied Science, BAEC, BSMMU Campus, Shahbag, Dhaka
\end{abstract}

\begin{abstract}
High dose I-131 therapy is administered to thyroid cancer patients after thyroidectomy. In the treatment process when a radiopharmaceutical is administered to patients, they become a mobile source of radiation. After the treatment, contamination may arise from large amount of activity, which is excreted during the period of hospitalization To reduce the rate of exposure it is essential to monitor workplace. This study determines the rate of radiation exposure in the room where the patient stays during the period of hospitalization as well as the adjacent rooms and surrounding areas.
\end{abstract}

\section{INTRODUCTION}

I-131, as sodium or potassium iodine, is supplied, either in liquid form or in a gelatin capsule. Iodine is extracted from the gut into the bloodstream and taken up by functioning thyroid tissue (including active metastasis). In malignant disease, where the patient has had a total or subtotal thyroidectomy prior to therapy, the uptake may be as low as $2 \%$ in residual thyroid tissue. The remainder is excreted primarily by the kidneys and consequently the patient should be encouraged to drink freely to minimize dose to kidneys, bladder and gonads (1). In most cases, $50-60 \%$ of the administered activity is excreted in the first 24 hours and around $85 \%$ over a stay of $4-5$ days in hospital. This represents a significant potential for radioactive contamination. The next most significant pathway is saliva. This will manifest in contamination of eating and drinking utensils and pillow coverings (due to saliva excretion during sleep). Lesser pathway are sweat and faces. Therefore it is essential to monitor the workplace to reduce the rate of exposure. This study determines the rate of radiation exposure in the room where the patient stays during the period of hospitalization as well as the adjacent rooms and surrounding areas.

Radiation precautions were taken to minimize the spread of contamination from urine, saliva and perspiration to hospital personnel, patients and visitors. The patients used private sanitary facilities, have meal using disposable table service and stored their linen in an isolated box for monitoring by 
trained radiation safety staff. External radiation exposure was minimized by adhering the therapy protocol suggested by SNMB (Society of Nuclear Medicine, Bangladesh)(2) and guidelines of BAERA (Bangladesh Atomic Energy Regulatory Authority) (3) that requires patients to stay in a private room until activity reduces to $1100 \mathrm{MBq}(\sim 30 \mathrm{mCi})$. Visitors and nursing staff could minimize exposure to radiation by reducing the time spent in close proximity of the patient and by increasing their distance from the patient.

\section{METHOD AND MATERIALS}

In this study, patients of DTC (Differentiate thyroid cancer) who received (30-250) mCi I-131 were observed for radiation exposure level. All these patients were given high dose of radioiodine 4-6 weeks after thyroid surgery. Among them, 17 patients were selected. Patients were admitted into single bed isolated cabins, used private sanitary facilities, had separate table/room service and their linen was stored in box for monitoring by trained radiation safety staff. A systematic survey of patients exposure rate was carried out over a period of 1 month using survey meter (Model Austral Rad Mini 8-in-1 \#1091 Australia) in different places of clinic like toilet, corridor and close to wall of next room, patient's bed in the next room at distances of 1,2 and 3 meter.

\section{RESULTS}

The exposure rate at the outer surface of door was different or fluctuated on the 1st day (Table -1). 
Table -1: Survey Meter Count for CA-Thyroid Patient in $\mu \mathrm{Sv} / \mathrm{h}$ A. Dose Recorded From Patients

\begin{tabular}{|c|c|c|c|c|c|c|c|c|c|c|c|c|c|c|c|c|}
\hline \multirow{2}{*}{$\begin{array}{l}\text { No. of } \\
\text { Patient }\end{array}$} & \multicolumn{3}{|c|}{$1^{\text {st }}$ Day } & \multicolumn{3}{|c|}{$2^{\text {nd }}$ Day } & \multicolumn{3}{|c|}{$3^{\text {rd }}$ Day } & \multicolumn{3}{|c|}{$4^{\text {th }}$ Day } & \multicolumn{3}{|c|}{$5^{\text {th }}$ Day } & \\
\hline & $1 \mathrm{M}$ & $2 \mathrm{M}$ & $3 \mathrm{M}$ & $1 \mathrm{M}$ & $2 \mathrm{M}$ & $3 \mathrm{M}$ & $1 \mathrm{M}$ & $2 \mathrm{M}$ & $3 \mathrm{M}$ & $1 \mathrm{M}$ & $2 \mathrm{M}$ & $3 \mathrm{M}$ & $1 \mathrm{M}$ & $2 \mathrm{M}$ & $3 \mathrm{M}$ & Activity \\
\hline 1 & 304 & 130 & 42 & 170 & 85 & 40 & 77 & 30 & 15 & 77 & 30 & 14 & 37.6 & 33.4 & 15 & $250 \mathrm{mCi}$ \\
\hline 2 & 265 & 111 & 95 & 76 & 29 & 13 & 7.7 & 2.7 & 2.1 & 2.6 & 1.0 & 0.8 & 2.03 & 1.04 & .08 & $100 \mathrm{mCi}$ \\
\hline 3 & 170 & 107 & 55 & 50 & 29 & 20 & 6.8 & 5.7 & 1.6 & 3.1 & 1.3 & 0.7 & 3.0 & 2.7 & 2.1 & $150 \mathrm{mCi}$ \\
\hline 4 & 60 & 50 & 29 & 16 & 10 & 8 & 15 & 4.9 & 1.9 & 16 & 5.5 & 7.0 & 7.03 & 6 & 4.6 & $75 \mathrm{mCi}$ \\
\hline 5 & 142 & 91 & 63 & 33 & 10 & 8 & 20 & 8.2 & 5 & 9 & 7.2 & 6.9 & 5.1 & 4.6 & 4.4 & $150 \mathrm{mCi}$ \\
\hline 6 & 55 & 46 & 24 & 16 & 10 & 8 & 5.0 & 6.8 & 9.2 & 2.0 & 2.7 & 3.6 & 3.0 & 2.5 & 1 & $30 \mathrm{mCi}$ \\
\hline 7 & 75 & 41 & 22 & 56 & 32 & 20.75 & 15.5 & 3.3 & 3.2 & 13.5 & 6.5 & 2.2 & 4.7 & 4 & 1.4 & $75 \mathrm{mCi}$ \\
\hline 8 & 125 & 70 & 50 & 111 & 38 & 20.9 & 10.9 & 5.4 & 2.5 & 9 & 5.2 & 3.8 & 3.2 & 2.7. & 2.1 & $100 \mathrm{mCi}$ \\
\hline 9 & 280 & 135 & 80 & 78.8 & 28 & 20 & 10.7 & 6.8 & 4.1 & 8.1 & 7.5 & 5.1 & 4.2 & 3.7 & 3.3 & $150 \mathrm{mCi}$ \\
\hline 10 & 160 & 75 & 30 & 150 & 57 & 28 & 17 & 13 & 5.5 & 15 & 12 & 4.7 & 12.3 & 9.5 & 7.1 & $150 \mathrm{mCi}$ \\
\hline 11 & 485 & 305 & 75 & 385 & 110 & 67 & 52 & 22 & 15 & 44 & 18 & 11 & 33.6 & 16.2 & 11.3 & $250 \mathrm{mCi}$ \\
\hline 12 & 156 & 92 & 41 & 95 & 22 & 11.2 & 12 & 4.3 & 3.5 & 10 & 3.2 & 2.1 & 11.3 & 9 & 7.6 & $75 \mathrm{mCii}$ \\
\hline 13 & 140 & 80 & 32 & 90 & 38 & 16 & 80 & 17 & 10 & 18 & 8 & 6 & 9.7 & 8.5 & 7.3 & $75 \mathrm{mCi}$ \\
\hline 14 & 190 & 65 & 27 & 88 & 32.11 & 16.98 & 50 & 20 & 10 & 18 & 9 & 6 & 9 & 8.06 & 6.8 & $100 \mathrm{mCi}$ \\
\hline 15 & 120 & 60 & 28 & 35.7 & 15.23 & 13.4 & 30.0 & 10.0 & 9.00 & 20 & 10 & 9 & 4.5 & 4.35 & 3.7 & $75 \mathrm{mCi}$ \\
\hline 16 & 200 & 150 & 83 & 55 & 35 & 19 & 30 & 16 & 10 & 10 & 6 & 4 & 2.9 & 2.4 & 2 & $100 \mathrm{mCi}$ \\
\hline 17 & 70 & 65 & 50 & 25 & 24 & 15 & 15 & 10 & 8 & 3 & 3 & 2 & 2.8 & 2.6 & 2.5 & $30 \mathrm{mCi}$ \\
\hline
\end{tabular}

\section{B. Dose Recorded From Toilet}

\begin{tabular}{|c|c|c|c|c|c|c|c|c|c|c|c|c|c|c|c|c|}
\hline \multirow{2}{*}{$\begin{array}{l}\text { No. of } \\
\text { Patient }\end{array}$} & \multicolumn{3}{|c|}{$1^{\text {st }}$ Day } & \multicolumn{3}{|c|}{$2^{\text {nd }}$ Day } & \multicolumn{3}{|c|}{$3^{\text {rd }}$ Day } & \multicolumn{3}{|c|}{$4^{\text {th }}$ Day } & \multicolumn{3}{|c|}{$5^{\text {th }}$ Day } & \multirow[b]{2}{*}{ Activity } \\
\hline & $1 \mathrm{M}$ & $2 \mathrm{M}$ & $3 \mathrm{M}$ & $1 \mathrm{M}$ & $2 \mathrm{M}$ & $3 \mathrm{M}$ & $1 \mathrm{M}$ & $2 \mathrm{M}$ & $3 \mathrm{M}$ & $1 \mathrm{M}$ & $2 \mathrm{M}$ & $3 \mathrm{M}$ & $1 \mathrm{M}$ & $2 \mathrm{M}$ & $3 \mathrm{M}$ & \\
\hline 1 & $\mathrm{x}$ & $\mathrm{x}$ & $\mathrm{x}$ & 110 & 101 & 98 & 20.1 & 16.4 & 9.3 & 13.3 & 7.6 & 3.3 & 10.5 & 7.09 & 2 & $250 \mathrm{mCi}$ \\
\hline 2 & $\mathrm{x}$ & $\mathrm{x}$ & $\mathrm{X}$ & 44 & 38 & 19 & 10.4 & 4.3 & 3.7 & 1.3 & 1.2 & 1.1 & 2.5 & 3.5 & 1.6 & $100 \mathrm{mCi}$ \\
\hline 3 & $\mathrm{x}$ & $\mathrm{x}$ & $\mathrm{x}$ & 22 & 17 & 13 & 16 & 13.1 & 10.2 & 9.4 & 7.5 & 3.2 & 5.4 & 4.4 & 1.8 & $150 \mathrm{mCi}$ \\
\hline 4 & $\mathrm{x}$ & $\mathrm{x}$ & $\mathrm{x}$ & 6 & 4.9 & - & 5.5 & 4.2 & - & 5.3 & 4.5 & - & 2.6 & 1.8 & - & $75 \mathrm{mCi}$ \\
\hline 5 & $\mathrm{x}$ & $\mathrm{x}$ & $\mathrm{x}$ & 25 & 16.7 & 13 & 11.5 & 7.3 & 5.1 & 6.2 & 4.1 & 2.6 & 3.8 & 3.4 & 2.3 & $150 \mathrm{mCi}$ \\
\hline 6 & $\mathrm{x}$ & $\mathrm{x}$ & $\mathrm{x}$ & 5.9 & 5.2 & 5 & 4.8 & 4.4 & 4 & 4 & 3.2 & 3 & 2 & 1 & 1 & $30 \mathrm{mCi}$ \\
\hline 7 & $\mathrm{x}$ & $\mathrm{x}$ & $\mathrm{x}$ & 10.7 & 6.1 & 5.0 & 7.2 & 6.4 & 5.7 & 5.1 & 3.3 & 2.9 & 2.6 & 2.3 & 2.1 & $75 \mathrm{mCi}$ \\
\hline 8 & $\mathrm{x}$ & $\mathrm{x}$ & $\mathrm{x}$ & 6.4 & 4.1 & 3.8 & 6.1 & 5.1 & 3.8 & 3.3 & 2.2 & 2 & 2.4 & 2.2 & 2.2 & $100 \mathrm{mCi}$ \\
\hline 9 & $\mathrm{x}$ & $\mathrm{x}$ & $\mathrm{x}$ & 7.9 & 7.7 & 7.4 & 5.2 & 3.3 & 2.4 & 4.9 & 4.5 & 2.2 & 3 & 2.5 & 2.3 & $150 \mathrm{mCi}$ \\
\hline 10 & $\mathrm{X}$ & $\mathrm{x}$ & $\mathrm{x}$ & 13 & 11 & - & 12 & 6.1 & - & 10 & 5.5 & - & 4.8 & 4.2 & - & $150 \mathrm{mCi}$ \\
\hline 11 & $\mathrm{x}$ & $\mathrm{x}$ & $\mathrm{x}$ & 33 & 25 & - & 24 & 20 & - & 9 & 7 & - & 9.2 & 7.7 & - & $250 \mathrm{mCi}$ \\
\hline 12 & $\mathrm{x}$ & $\mathrm{x}$ & $\mathrm{x}$ & 11.6 & 8.8 & 7.3 & 7.4 & 5.3 & 4.2 & 4.1 & 3.3 & 2.1 & 5 & 4.2 & 3.3 & $75 \mathrm{mCi}$ \\
\hline 13 & $\mathrm{x}$ & $\mathrm{x}$ & $\mathrm{x}$ & 7.64 & 6.44 & 5.63 & 6.40 & 5.2 & 4.2 & 5.7 & 4.2 & 0.22 & 5.3 & 4.3 & 2.8 & $75 \mathrm{mCi}$ \\
\hline 14 & $\mathrm{x}$ & $\mathrm{x}$ & $\mathrm{x}$ & 8.55 & 5.45 & 4.7 & 7 & 5 & 5 & 3.7 & 3 & 2.25 & 4.9 & 4.1 & 3.5 & $100 \mathrm{mCi}$ \\
\hline 15 & $\mathrm{x}$ & $\mathrm{x}$ & $\mathrm{x}$ & 7.3 & 4.8 & 6.6 & 5.25 & 4.1 & 4.6 & 3 & 2 & 2 & 3.3 & 3 & 2.7 & $75 \mathrm{mCi}$ \\
\hline 16 & $\mathrm{x}$ & $\mathrm{x}$ & $\mathrm{x}$ & 7.9 & 5 & 4.25 & 5.5 & 3.2 & 3 & 4 & 3 & 2 & 3.4 & 3 & 2.9 & $100 \mathrm{mCi}$ \\
\hline 17 & $\mathrm{x}$ & $\mathrm{x}$ & $\mathrm{x}$ & 7.9 & 5 & 4.25 & 5.5 & 3.2 & 3 & 4 & 3 & 2 & 3.4 & 3 & 2.9 & $30 \mathrm{mCi}$ \\
\hline
\end{tabular}

\section{Dose Recorded At Corridor from Door (door must be closed)}

\begin{tabular}{|c|c|c|c|c|c|c|c|c|c|c|c|c|c|c|c|c|}
\hline \multirow{2}{*}{$\begin{array}{l}\text { No. of } \\
\text { Patient }\end{array}$} & \multicolumn{3}{|c|}{$1^{\text {st }}$ Day } & \multicolumn{3}{|c|}{$2^{\text {nd }}$ Day } & \multicolumn{3}{|c|}{$3^{\text {rd }}$ Day } & \multicolumn{3}{|c|}{$4^{\text {th }}$ Day } & \multicolumn{3}{|c|}{$5^{\text {th }}$ Day } & \multirow[b]{2}{*}{ Activity } \\
\hline & $1 \mathrm{M}$ & $2 \mathrm{M}$ & $3 \mathrm{M}$ & $1 \mathrm{M}$ & $2 \mathrm{M}$ & $3 \mathrm{M}$ & $1 \mathrm{M}$ & $2 \mathrm{M}$ & $3 \mathrm{M}$ & $1 \mathrm{M}$ & $2 \mathrm{M}$ & $3 \mathrm{M}$ & $1 \mathrm{M}$ & $2 \mathrm{M}$ & $3 \mathrm{M}$ & \\
\hline 1 & 9.88 & 7 & 5 & 7.8 & 5.4 & 3.4 & 2.4 & 1.7 & 1.4 & 2.4 & 1.7 & 1.3 & 1.8 & 1.6 & 1.0 & $250 \mathrm{mCi}$ \\
\hline 2 & 9.8 & 8 & 7.9 & 2.44 & 1.9 & 1.39 & 0.84 & 0.7 & 0.17 & 0.17 & 0.16 & 0.15 & 0.15 & 0.14 & 0.13 & $100 \mathrm{mCi}$ \\
\hline 3 & 11 & 10 & 16 & 9 & 6.0 & 12 & 8 & 5 & 9.7 & 5 & 3 & 7 & 3 & 3 & 3 & $150 \mathrm{mCi}$ \\
\hline 4 & 7 & 6 & 9 & 6 & 5 & 8 & 3 & 4 & 6 & 2.1 & 2.7 & 5 & 1.1 & 1.01 & 2 & $75 \mathrm{mCi}$ \\
\hline 5 & 11 & 14 & 9 & 9.4 & 4.3 & 2.4 & 3.2 & 3.8 & 1.5 & 3.0 & 3.8 & 1.3 & 0.9 & 0.6 & 0.22 & $150 \mathrm{mCi}$ \\
\hline 6 & 3.4 & 2.9 & 2.4 & 2.9 & 2.4 & 2.4 & 2 & 2.2 & 1.9 & 1.8 & 1.5 & 1.6 & 1 & 1 & 0.09 & $30 \mathrm{mCi}$ \\
\hline 7 & 6.6 & 4.4 & 3 & 8.3 & 10.04 & 6.6 & 3.2 & 6.6 & 1.5 & 0.03 & 0.03 & 0.01 & 1.75 & 11.75 & 2.0 & $75 \mathrm{mCi}$ \\
\hline 8 & 18 & 8 & 6 & 7.6 & 3.1 & 1.3 & 1.5 & 0.20 & 0.15 & 0.22 & 0.20 & 0.22 & 0.53 & 0.63 & 0.25 & $100 \mathrm{mCi}$ \\
\hline 9 & 12 & 9 & 8 & 3.9 & 4 & 3.1 & 1.0 & 0.6 & 0.2 & 1.29 & 1.6 & 0.53 & 0.9 & 0.8 & 0.72 & $150 \mathrm{mCi}$ \\
\hline 10 & 7 & 6 & 4 & 7 & 5.8 & 4.8 & 4.6 & 4.4 & 4.3 & 1.1 & 0.34 & 0.17 & 2.3 & 1.9 & 1.6 & $150 \mathrm{mCi}$ \\
\hline 11 & 15 & 12 & 16 & 11 & 11 & 8.8 & 7.7 & 4.1 & 3.3 & 2.5 & 2.2 & 1.7 & 7.4 & 6.7 & 5.3 & $250 \mathrm{mCi}$ \\
\hline 12 & 12 & 7 & 5 & 3.2 & 2.7 & 2.1 & 2.1 & 2.1 & 1.9 & 1.2 & 0.35 & 0.22 & 1.25 & 1 & 0.9 & $75 \mathrm{mCi}$ \\
\hline 13 & 13 & 6 & 4 & 4.5 & 1.9 & 0.44 & 4 & 0.92 & 0.30 & 1.5 & 1.3 & 0.75 & 3.8 & 2.3 & 2.2 & $75 \mathrm{mCi}$ \\
\hline 14 & 7 & 5 & 4 & 3.30 & 2.53 & 1.96 & 2.10 & 2.00 & 1.17 & 1 & 1 & 1 & 1.2 & 1.1 & 0.63 & $100 \mathrm{mCi}$ \\
\hline 15 & 7 & 6 & 5 & 3.3 & 2.54 & 1.96 & 2.10 & 1.92 & 1 & 2 & 1.1 & 1 & 1.2 & 1.1 & 0.63 & $75 \mathrm{mCi}$ \\
\hline 16 & 12 & 7 & 5 & 7 & 5 & 3 & 5 & 4 & 2 & 4 & 3 & 2 & 0.8 & 0.8 & 0.3 & $100 \mathrm{mCi}$ \\
\hline 17 & 10 & 7 & 6 & 7 & 5 & 3 & 6 & 4 & 2 & 5 & 3 & 2 & 0.8 & 0.8 & 0.3 & $30 \mathrm{mCi}$ \\
\hline
\end{tabular}




\section{In the next Room}

\begin{tabular}{|c|c|c|c|c|c|c|c|c|c|c|c|c|c|c|c|c|}
\hline \multirow[b]{2}{*}{$\begin{array}{c}\text { No. } \\
\text { of } \\
\text { Patient }\end{array}$} & \multicolumn{3}{|c|}{$1^{\text {st }}$ Day } & \multicolumn{3}{|c|}{$2^{\text {nd }}$ Day } & \multicolumn{3}{|c|}{$3^{\text {rd }}$ Day } & \multicolumn{3}{|c|}{$4^{\text {th }}$ Day } & \multicolumn{3}{|c|}{$5^{\text {th }}$ Day } & \multirow[b]{2}{*}{ Activity } \\
\hline & $\begin{array}{l}\text { Close } \\
\text { to } \\
\text { Wall }\end{array}$ & $\begin{array}{l}\text { At } \\
\text { Pt. } \\
\text { Bed }\end{array}$ & $\begin{array}{l}\text { Middle } \\
\text { of } \\
\text { Room }\end{array}$ & $\begin{array}{l}\text { Close } \\
\text { to } \\
\text { Wall }\end{array}$ & $\begin{array}{l}\text { At } \\
\text { Patient } \\
\text { Bed }\end{array}$ & $\begin{array}{l}\text { Middle } \\
\text { of } \\
\text { Room }\end{array}$ & $\begin{array}{l}\text { Close } \\
\text { to } \\
\text { Wall }\end{array}$ & $\begin{array}{l}\text { At } \\
\text { Pt } \\
\text { Bed }\end{array}$ & $\begin{array}{l}\begin{array}{l}\text { Middle } \\
\text { of } \\
\text { Room }\end{array} \\
\end{array}$ & $\begin{array}{l}\text { Close } \\
\text { to } \\
\text { Wall }\end{array}$ & $\begin{array}{l}\text { At Pt. } \\
\text { Bed }\end{array}$ & $\begin{array}{l}\text { Middle } \\
\text { of } \\
\text { Room }\end{array}$ & $\begin{array}{l}\text { Close } \\
\text { to } \\
\text { Wall }\end{array}$ & $\begin{array}{l}\text { At Pt } \\
\text { Bed }\end{array}$ & $\begin{array}{l}\text { Middle } \\
\text { of } \\
\text { Room }\end{array}$ & \\
\hline 1 & 2 & 1.6 & 1 & 0.345 & 0.299 & 0.189 & 0.25 & 0.16 & 0.17 & 0.25 & 0.3 & 0.22 & 0.34 & 0.02 & 0.22 & $250 \mathrm{mCi}$ \\
\hline 2 & 8.1 & 8 & 7.9 & 3.59 & 1.67 & 0.175 & 1.5 & 1.1 & 0.5 & 1.8 & 0.7 & 0.3 & 1.1 & 0.6 & 0.03 & $100 \mathrm{mCi}$ \\
\hline 3 & 3 & 2 & 2 & 1 & 0.34 & 0.7 & 6.0 & 4.1 & 2.9 & 0.44 & 0.34 & 0.22 & 0.34 & 0.25 & 0.01 & $150 \mathrm{mCi}$ \\
\hline 4 & 2 & 1.9 & 1.8 & 1.7 & 1.6 & 1.4 & 1 & 1 & 1 & 0.86 & 0.65 & 0.54 & 0.22 & 0.22 & 0.25 & $75 \mathrm{mCi}$ \\
\hline 5 & 3.5 & 2 & 2.5 & 3 & 1.8 & 2 & 2 & 1 & 1.3 & 1.4 & 1 & 1 & 0.86 & 0.24 & 0.55 & $150 \mathrm{mCi}$ \\
\hline 6 & 3 & 2.2 & 2.5 & 2 & 1.5 & 1.8 & 2 & 1.1 & 1.5 & 1.1 & 1 & 1 & 0.77 & 0.36 & 0.68 & $30 \mathrm{mCi}$ \\
\hline 7 & 2.5 & 1.9 & 1.5 & 0.72 & 0.63 & 0.7 & 1.0 & 1.1 & 1.2 & 0.25 & 0.25 & 0.3 & 1.25 & 0.22 & 0.2 & $75 \mathrm{mCi}$ \\
\hline 8 & 2.5 & 1.9 & 1.5 & 1.8 & 1.6 & 1.3 & 1.3 & 1.1 & 1.1 & 1.1 & 1.0 & 0.17 & 0.22 & 0.2 & 0.175 & $100 \mathrm{mCi}$ \\
\hline 9 & 16 & 7 & 5 & 4.7 & 3.9 & 3.2 & 3.7 & 2.9 & 2.4 & 0.17 & 0.25 & 0.17 & 1.3 & 1 & 0.9 & $150 \mathrm{mCi}$ \\
\hline 10 & 2 & 16 & 1 & 2.5 & 2.3 & 1.9 & 1.4 & 1.3 & 2.0 & 1.7 & 0.25 & 0.17 & 0.5 & 0.34 & 0.22 & $150 \mathrm{mCi}$ \\
\hline 11 & 5 & 4 & 4 & 6.7 & 5.4 & 4.4 & 3.9 & 2.3 & 1.8 & 1.5 & 0.02 & 0.2 & 1.4 & 13 & 1.3 & $250 \mathrm{mCi}$ \\
\hline 12 & 2.2 & 1 & 0.5 & 5.1 & 3.6 & 2.7 & 5.5 & 3.2 & 2.1 & 0.8 & 0.6 & 0.45 & 0.53 & 0.44 & 0.34 & $75 \mathrm{mCi}$ \\
\hline 13 & 2 & 1 & 0.9 & 6.4 & 2.7 & 1.58 & 0.40 & 1.60 & 1.30 & 0.3 & 0.2 & 0.2 & 1.7 & 1.58 & 1.20 & $75 \mathrm{mCi}$ \\
\hline 14 & 3.5 & 2.6 & 1.7 & 3.11 & 2.15 & 1.58 & 2.10 & 1.80 & 1.00 & 1 & 1 & 0.7 & 0.72 & 0.53 & 0.44 & $100 \mathrm{mCi}$ \\
\hline 15 & 0.3 & 0.29 & 0.29 & 0.44 & 0.29 & 0.53 & 0.20 & 0.17 & 0.11 & 0.20 & 0.20 & 0.20 & 0.22 & 0.22 & 0.20 & $75 \mathrm{mCi}$ \\
\hline 16 & 2 & 1 & 1 & 0.75 & 0.22 & 0.22 & 0.15 & 0.10 & 0.10 & 0.17 & 0.12 & 0.12 & 1.5 & 1.2 & 1.1 & $100 \mathrm{mCi}$ \\
\hline 17 & 1 & 1 & 1 & 0.75 & 0.5 & 0.1 & 0.5 & 0.3 & 0.1 & 0.17 & 0.12 & 0.12 & 1.5 & 1.2 & 1.1 & $30 \mathrm{mCi}$ \\
\hline
\end{tabular}

Dose rate varied between $(18$ - 3.4) $\mu \mathrm{Sv} / \mathrm{h}$. The dose rate in the toilet varied between (13-5.9) $\mu \mathrm{Sv} / \mathrm{h}$. Among them, few patients dose rate in the toilet was found high (110-22) $\mu \mathrm{Sv} / \mathrm{h}$. Dose rate varied between $(16-0.3) \mu \mathrm{Sv} / \mathrm{h}$ in next room walls, close to the patient's room.

\section{DISCUSSION}

The exposure rate at the outer surface of door was different or fluctuated on the 1st day (Table -1). Dose rate varied between (18- $3.4 \mu \mathrm{Sv} / \mathrm{h})$ due to difference in patients' activities, position of rooms, and position of patients' bed. Dose rate was high when a patient's bed was near the door and when the patient was sitting in the veranda the dose rate was low. Besides, when two patients stayed in opposite rooms with common corridor, the corridor reading was high and fluctuated. Therefore, changing the room arrangement can minimize radiation level in corridors. The dose rate in the toilet varied between $(13-5.9 \mu \mathrm{Sv} / \mathrm{h})$, when the patient took sufficient fluid like water, soft drinks, soup etc. and proper flush of the toilet. Dose rate in the toilet was found high $(110-22 \mu \mathrm{Sv} / \mathrm{h})$ due to intake insufficient fluid like water, soft drinks, soup etc. In some toilets, there were not enough space to measure exposure rate at $3 \mathrm{~m}$ distances. In the next room walls, close to the patient's room, dose rate varied between 0.3 $\mu \mathrm{Sv} / \mathrm{h} 16 \mu \mathrm{Sv} / \mathrm{h}$. Dose rate of wall close to patient's bed was high for one patient due to the patients bed in one room is placed against the same wall as a bed in an adjacent room. It is to be ensured that the patient's bed in one room is not placed against the same wall as a bed in an adjacent room. Otherwise, the distance between the beds will also be very small and the walls do not provide effective protection against this type of radiation (4). When a cabin is reserved for a patient's admission it should be noted that no child patient or pregnant women 
is admitted into the next room as these groups of patients' embryos, fetuses and children are at greatest risk from external radiation exposure $(5,6)$. After 5 days of hospitalization, the radiation level did not exceed maximum permissible limit (7), in fact it was significantly below the permissible limit

\section{CONCLUSION}

Radiation is an essential part of daily life. Not only radiation is an essential part of human life, it can also be used to improve human life. It is the role of health care providers to use ionization radiation in medicine efficiently to maximize benefit and minimize risk. Survey of the radiation exposure is required limit unnecessary radiation exposure.

\section{REFERENCES}

1. IAEA Radiation Protection of Patients (RPOP) Specific Therapies, Iodine-131 used in thyroid cancer. Website:https://rpop.iaea.org/RPoP/Content/InformationFor/HealthPro

2. Faridul Alam, A S M Moinul Islam, M A Karim. Therapy protocol for thyroid carcinoma and thyrotoxicosis with I-131 Adopted in the National Workshop on Management of Thyroid Cancer and Thyrotoxicosis by Nuclear Medicine Technique A Concensious Report. Bangladesh J.Nucl. Med. 2002; 5(2):39-42.

3. Regulatory Guide on Radiation Protection in Nuclear Medicine. Regulatory Guide Series No.NSRC-NM-G-01.NSRCD, BAEC, December 2002,p-39.

4. Radiation Protection 97.Radiation Protection following I-131 therapy. Directorate General Environment, Nuclear Safety and Civil Protection, European Commission.1998: p-14.

5. NCRP. Protection in Nuclear Medicine and Ultrasound Diagnostic procedures in Children, National Council on Radiation, Protection and Measurements, Washington, DC, Report No. 73, 1983: 2.

6. Committee on Biological Effects of Ionizing Radiation, National Research Council (BEIR). The effects on population of Exposure of low level of Ionizing Radiation. Washington, DC National Academy Press: 1980.

7. ANZAPNM Basic Science Course, AS 2243.4 safety in Laboratories Part \$: Ionizing Radiation, 1998. 